\title{
Functional link between mitochondria and Rnr3, the minor catalytic subunit of yeast ribonucleotide reductase
}

\author{
Isaac Corcoles-Saez ${ }^{1}$, Jean-Luc Ferat ${ }^{2}$, Michael Costanzo ${ }^{3}$, Charles M. Boone ${ }^{3}$ and Rita S. Cha ${ }^{1, *}$ \\ ${ }^{1}$ School of Medical Sciences, North West Cancer Research Institute, Bangor University, Deniol Road, Bangor, LL57 2UW, United \\ Kingdom. \\ 2 Institute of Integrative Biology of the Cell (I2BC), Avenue de la Terrasse, Paris, France. \\ 3 University of Toronto, Donnelly Centre, 160 College Street, Toronto, Ontario, M5S 3E1, Canada.. \\ * Corresponding Author: \\ Rita S. Cha, Tel: +44 (0)1248 38286; E-mail: r.cha@bangor.ac.uk
}

\begin{abstract}
Ribonucleotide reductase (RNR) is an essential holoenzyme required for de novo synthesis of dNTPs. The Saccharomyces cerevisiae genome encodes for two catalytic subunits, Rnr1 and Rnr3. While Rnr1 is required for DNA replication and DNA damage repair, the function(s) of Rnr3 is unknown. Here, we show that carbon source, an essential nutrient, impacts Rnr1 and Rnr3 abundance: Non-fermentable carbon sources or limiting concentrations of glucose down regulate Rnr1 and induce Rnr3 expression. Oppositely, abundant glucose induces Rnr1 expression and down regulates Rnr3. The carbon source dependent regulation of Rnr3 is mediated by Mec1, the budding yeast ATM/ATR checkpoint response kinase. Unexpectedly, this regulation is independent of all currently known components of the Mec1 DNA damage response network, including Rad53, Dun1, and Tel1, implicating a novel Mec1 signalling axis. $r n r 3 \Delta$ leads to growth defects under respiratory conditions and rescues temperature sensitivity conferred by the absence of Tom6, a component of the mitochondrial TOM (translocase of outer membrane) complex responsible for mitochondrial protein import. Together, these results unveil involvement of Rnr3 in mitochondrial functions and Mec1 in mediating the carbon source dependent regulation of Rnr3.
\end{abstract}

doi: $10.15698 /$ mic2019.06.680 Received originally: 24.12.2019; in revised form: 09.05.2019, Accepted 13.05.2019, Published 20.05.2019.

Keywords: Rnr1, Rnr3, Mec1, carbon source, respiration, mitochondria, dNTP.
Abbreviations:
$D D R-D N A$ damage response,
$G O$ - gene ontology,
$R N R$ - ribonucleotide reductase,
$S G A$ - synthetic genetic array,
TOM - translocase of outer
membrane,
WGD - whole genome duplication.

\section{INTRODUCTION}

Ribonucleotide reductase (RNR) is a conserved holoenzyme required for de novo synthesis of dNTPs, the building blocks of DNA [1]. The eukaryotic RNR is a tetrameric complex composed of two large R1 catalytic subunits and two small R2 regulatory subunits. The R1 subunit contains an allosteric regulatory site that monitors the intracellular ATP/dATP ratio and controls the overall RNR catalytic activity. The R2 subunit contains a critical tyrosine residue where the radical transfer to the active site in the $\mathrm{R} 1$ subunit initiates [1, 2].

The genome of Saccharomyces cerevisiae encodes for four RNR proteins; Rnr1 and Rnr3 for the catalytic R1 subunits and Rnr2 and Rnr4 for the regulatory R2 subunits [3]. While expression of Rnr2 and Rnr4 is constitutive, Rnr1 is induced specifically during $\mathrm{S}$ phase. Rnr3, on the other hand, is detectable only under the condition of DNA damage or replication stress [3].
The budding yeast Mec1 is an essential serine/threonine kinase, responsible for mediating the genotoxic stress dependent induction of Rnr3 [4]. Mec1 is an ATM/ATR protein, a family of conserved phosphatidylinositol 3-kinase like kinases (PIKKs) best understood for their roles in mediating the DNA damage response (DDR) [5]. In addition, ATM/ATR proteins play critical roles in a number of fundamental processes, such as DNA replication, meiotic recombination, neuronal vesicle trafficking, and protein homeostasis [6-10]

In response to genotoxic stress, Mec1 activates RNR via two downstream kinases Rad53 and Dun1 [11]. Rad53 is an essential kinase that shares $24 \%$ and $30 \%$ identity with the human CHK1 and CHK2 kinases, respectively. The latter are ATM/ATR targets, which become activated in response to replication stress and DNA damage, respectively [5, 12]. Rad53 is phosphorylated in response to both replication stress (i.e like CHK1) and DNA damage (i.e. like CHK2) in a 
Mec1 dependent manner. Upon activation, Rad53 phosphorylates Dun1, which in turn activates RNR by inhibiting activities of three negative regulators; (i) Sml1, an allosteric inhibitor of Rnr1, (ii) Dif1, involved in nuclear transport of Rnr2 and Rnr4, and (iii) Rfx1/Crt1, a transcriptional repressor that downregulates $R N R 3$ expression [11, 13, 14].

$R N R 1$ and $R N R 2$ are essential in most yeast strain backgrounds, while inactivation of Rnr4 impacts genome duplication, DNA damage repair, and resistance to genotoxic stress. In contrast, $r n r 3 \Delta$ does not confer any obvious phenotypes, including sensitivity to replication stress or DNA damage despite the fact that its expression is massively induced during the DDR [15]. As such, the function(s) of Rnr3 remains elusive. Here, we present evidence for involvement of Rnr3 in a dNTP independent mitochondrial function(s).

\section{RESULTS}

\section{Carbon source dependent regulation of Rnr1 and Rnr3}

$R N R 1$ and RNR3 are paralogs that arose from the whole genome duplication (WGD) [16]. An outcome of the duplication was increased glycolytic flux, enabling the post WGD yeasts to meet the demands for ATP independently of mitochondrial respiration or oxidative phosphorylation [17]. This suggests that Rnr1 and Rnr3 might have a metabolism dependent function(s), and that their expression might be controlled by metabolic state of the cell. To address this possibility, we assessed the impact of two different carbon sources, glucose and glycerol, on Rnr1 and Rnr3 expression. Glucose is a fermentable carbon source, which at a standard concentration (2\%), promotes rapid fermentative proliferation. Glycerol is a non-fermentable carbon source metabolized via oxidative phosphorylation in the mitochondria. As reported [15], only Rnr1 was observed in a standard synthetic complete medium supplemented with $2 \%$ glucose (SCD) while Rnr3 remained undetectable (Figure 1A). The cells were collected and released into either fresh SCD or synthetic complete medium supplemented with $2 \%$ glycerol (SCG) and subjected to further incubation. Cells in SCD continued to divide (Figure $\mathbf{1 C}$ ). In contrast, the cells transferred to SCG ceased to divide for the first 24 hours (Figure 1C). During this period, Rnr1 abundance declined steadily until it became undetectable (Figure 1B). Cells began dividing again between 24 and 48 hours (Figure 1C). Remarkably, the resumption of cell division was accompanied by an increase in Rnr3 levels while Rnr1 remained undetectable (Figure 1B).

To determine whether the apparent Rnr1-to-Rnr3 switch was due to glycerol per se or its impact on cellular metabolism, we tested the effects of ethanol, another nonfermentable carbon source, and limiting concentrations $(0.1 \%)$ of glucose, which activate respiratory metabolism.
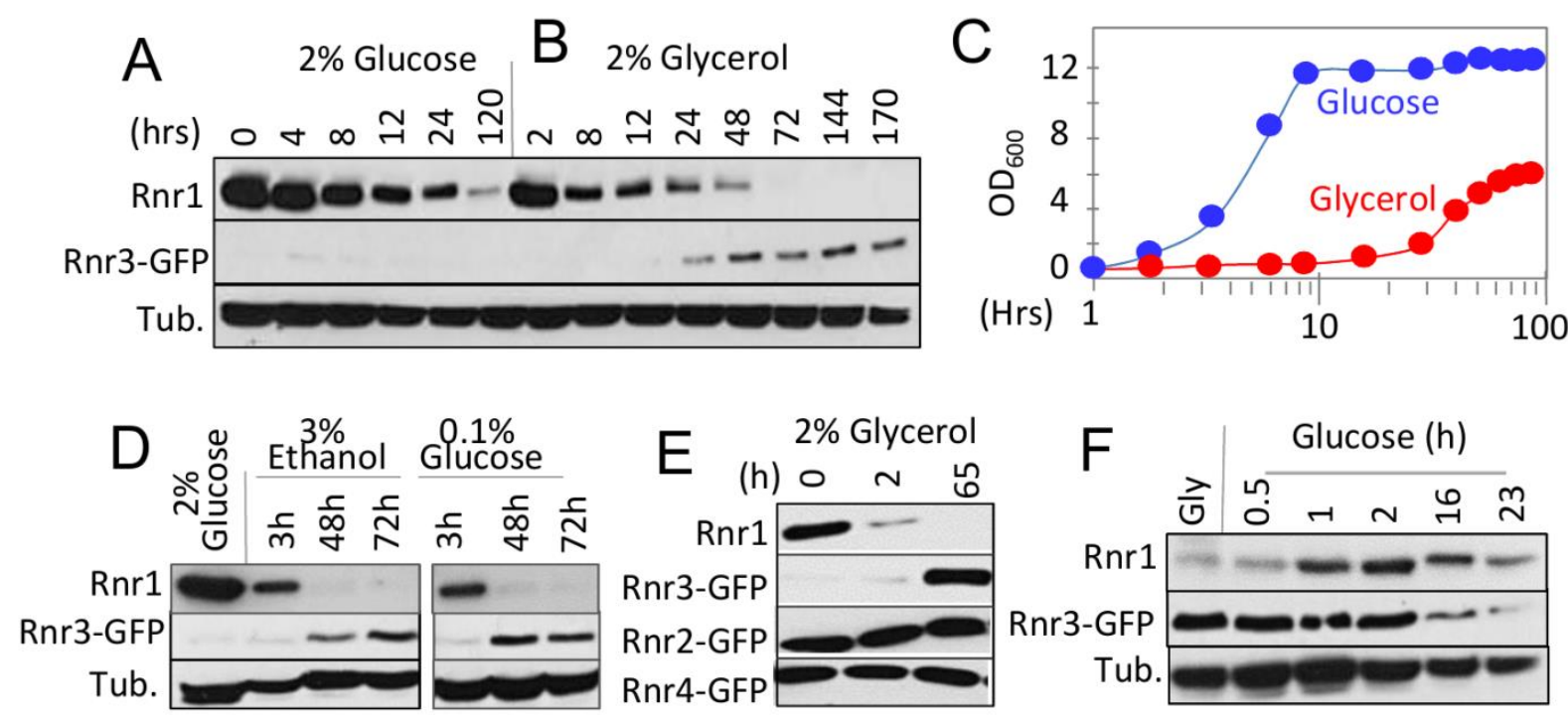

FIGURE 1: Impact of carbon source on the steady state levels of Rnr1 and Rnr3. (A, B) RNR3-GFP cells were grown to mid-log phase in synthetic complete medium supplemented with $2 \%$ glucose (SCD). The cells were collected and released into either SCD (A) or SC medium supplemented with $2 \%$ glycerol (SCG; B) for further incubation. Whole cell extract (WCE) samples were prepared at the indicated time points and subjected to Western blot analysis using $\alpha$-Rnr1 and $\alpha$-GFP antibodies. Tubulin was used as a loading control. (C) OD 600 of the SCD (blue) and SCG (red) cultures in panels A and B at the indicated time points. (D) RNR3-GFP cells were grown to mid-log phase in SCD. The cells were collected and released into SC medium supplemented with either $3 \%$ ethanol or $0.1 \%$ glucose for further incubation. WCE samples were prepared at the indicated time points and subjected to Western blot analysis using $\alpha$-Rnr 1 and $\alpha$-GFP antibodies. Tubulin was used as a loading control. (E) RNR2-GFP, RNR3-GFP, and RNR4-GFP strains were subjected to the glucose-to-glycerol medium switch as described in panel B. WCE samples were prepared at the indicated time points and subjected to Western blot analysis using $\alpha$-Rnr1 and $\alpha$ GFP antibodies. WCE preparation from the RNR3-GFP culture was used for $\alpha$-Rnr1 Western blot. (F) RNR3-GFP cells were grown to mid log phase in SCG. Cells were collected and released into SCD. WCE samples were prepared at the indicated time points and subjected to Western blot analysis using $\alpha$-Rnr1 and $\alpha$-GFP antibodies. Tubulin was used as a loading control. 
Results show that both induced Rnr1 down regulation and Rnr3 induction (Figure 1D). We infer that the Rnr1-to-Rnr3 switch is a cellular response to respiratory metabolism. We also tested the impact of carbon sources on Rnr2 and Rnr4, the two regulatory subunits of RNR. In contrast to Rnr1 and $\mathrm{Rnr}$, their expression was unaffected (Figure 1E).

The fact that Rnr1 expression is dominant over Rnr3 in glucose medium (e.g. Figure 1A) implies that the effects of respiratory carbon source are reversible. To test this, we performed a reciprocal medium switch experiment, from $2 \%$ glycerol to $2 \%$ glucose. Results show a notable increase in Rnr1 levels by 1 hour following the switch. And by 16 hours, a reduction in Rnr3 is also apparent (Figure 1F).

Taken together, these results unveil a carbon source dependent regulation of the two catalytic subunits of budding yeast RNR. Non-fermentable carbon sources or limiting concentrations of glucose, which activate respiratory metabolism, down regulate Rnr1 and induce Rnr3 expression. Oppositely, abundant glucose, which facilitates rapid fermentative proliferation, induces Rnr1 expression and down regulates $\mathrm{Rnr} 3$.

\section{Mec1 mediates both the carbon source dependent induc- tion and down regulation of Rnr3}

As mentioned above, Mec1 mediates the DDR dependent induction of Rnr3 $[11,13]$. To test its involvement in the carbon source dependent regulation, we assessed the impact of a kinase dead allele, mec1-kd, which remains viable in a sml1s suppressor mutation background [18]. WT, $m e c 1-k d s m / 1 \Delta$, and $s m / 1 \Delta$ strains were cultured in rich $2 \%$ glucose (YPD) or $2 \%$ glycerol (YPG) medium until they were in mid-log phase. Protein-extracts were prepared from each culture and analyzed by Western blot using antibodies against Rnr1 and Rnr3-GFP (Figure 2A). Glycerol dependent Rnr3 expression was abolished in the mec1-kd $s m / 1 \triangle$ strain, indicating that it was MEC1 dependent (Figure $2 \mathrm{~A}$ ). In contrast, $M E C 1$ was dispensable for the carbon source dependent down regulation of Rnr1 (Figure 2A).

Next, we wished to address whether Mec1 might also mediate the glucose dependent down regulation of Rnr3 (e.g. Figure 1F). However, because the glycerol dependent Rnr3 expression is absent in mec1-kd sm/1 $1 \Delta$ cells (Figure 2A), the strain would not be suitable for the experiment. Instead, we utilized a separation of function allele, mec1-4, which is viable but confers sensitivity to DNA damage, replication stress, as well as to elevated temperature [8]. This strain, in contrast to the mec1-kd $s m / 1 \Delta$, is proficient in glycerol dependent Rnr3 induction at $25^{\circ} \mathrm{C}$, a permissive temperature (Figure $\mathbf{2 C}$ ).

WT and mec1-4 cells in YPD were released into YPG and subjected to further incubation at $25^{\circ} \mathrm{C}, 33^{\circ} \mathrm{C}$ and $37^{\circ} \mathrm{C}$, a permissive, semi-permissive, and restrictive temperature for mec1-4, respectively. Robust increase in Rnr1 was observed by 3 hours in both the WT and mec1- 4 strains, irrespective of the temperature (Figure 2B, C). By 22 hours, a notable reduction in Rnr3 was observed in the mec1-4 at $25^{\circ} \mathrm{C}$, but not at $37^{\circ} \mathrm{C}$, a restrictive temperature for mec1- 4
(Figure $\mathbf{2 C}$ ). The latter shows that the glucose dependent down regulation of Rnr3 is also mediated by Mec1.

Carbon source dependent Rnr3 induction is independent of the DNA damage response network

Rnr3 expression during the DDR is mediated by the Mec1Rad53-Dun1 signaling axis (Figure 3A) [11]. To address involvement of the latter in the carbon source dependent Rnr3 induction, we tested the impact of rad53-K277A, a kinase dead allele, and dun $1 \Delta$. Results show that neither mutation had an impact on the glycerol dependent Rnr3 induction, despite the fact that both abolished the $\mathrm{HU}$ and MMS dependent induction (Figure 3B; red box). This indicates that Mec1 mediates the carbon source dependent
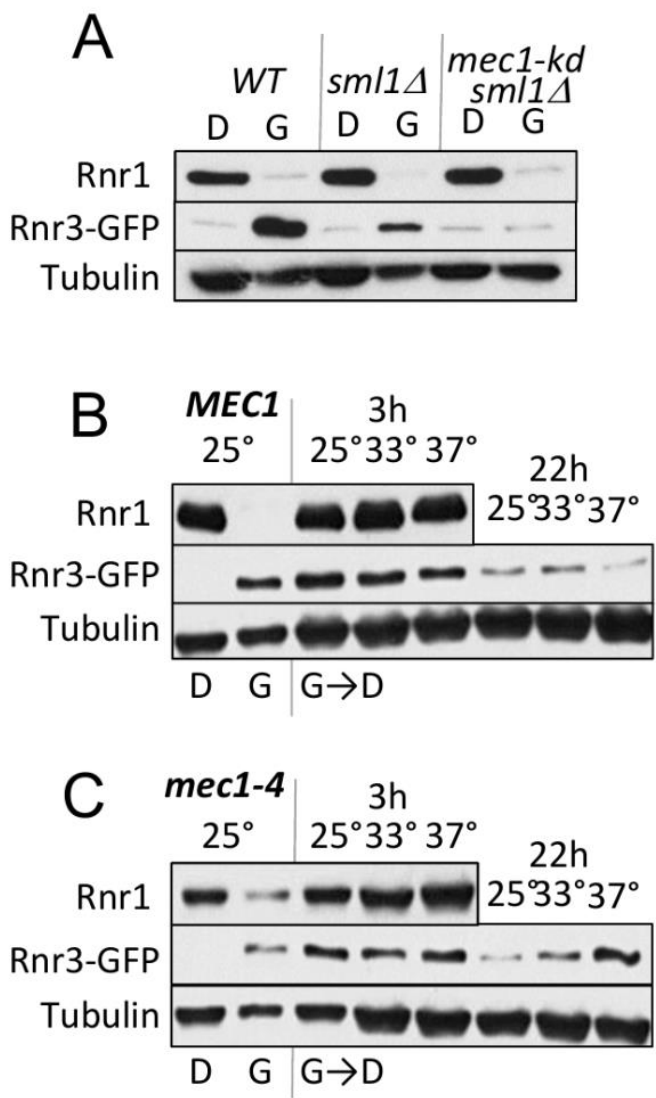

FIGURE 2: Mec1 promotes the carbon source dependent regulation of Rnr3. (A) $W T, s m / 1 \Delta$, and mec1-kd sm/1 $\Delta$ cells were grown to mid-log phase in YPD (“D”) or YPG (“G"). WCE samples were prepared and subjected to Western blot analysis using $\alpha$ Rnr1 and $\alpha$-GFP antibodies. Tubulin was used as a loading control. (B, C) WT and mec1-4 cells were grown to mid log phase in YPD (D) or YPG (G) at $25^{\circ} \mathrm{C}$, a permissive temperature for mec1-4 [8]. WCE samples were prepared and subjected to $\alpha$-Rnr1 and $\alpha$-GFP Western blot analysis. "G $\rightarrow D$ ": Cells grown to mid-log phase in YPG were collected and released into YPD and incubated at three different temperatures, $25^{\circ} \mathrm{C}, 30^{\circ} \mathrm{C}$, and $37^{\circ} \mathrm{C}$. WCE samples were prepared at 3- and 22- hours after the medium switch and subjected to Western blot analysis using $\alpha-R n r 1$ and $\alpha$-GFP antibodies. Tubulin was used as a loading control. 
Rnr3 induction independently of Rad53 and Dun1. In further support, we find that Rad53 remains unphosphorylated in response to glycerol (Figure $3 \mathrm{C}$ ).

We expanded the analysis to additional components of the Mec1 DDR network: Dif1, Sml1, Swi6, Mbp1, Ixr1, Crt10, Rfx1, Tel1 and Chk1 (Figure 3A)[19]. Dif1, Sml1, and Rfx1 are regulators of $\mathrm{Rnr} 2 / 4, \mathrm{Rnr} 1$, and $\mathrm{Rnr} 3$, respectively (above). Tel1 is another budding yeast ATM/ATR protein, which can partially substitute for Mec1 [20]. Chk1 is a serine/threonine kinase that functions to prevents anaphase onset [21]. And Swi6, Mbp1, Ixr1, and Crt10 are involved in regulation of $R N R 1$ and $R N R 3$ transcription respectively [19]. Results show that all of the tested mutants are proficient in glycerol dependent Rnr3 induction (Figure 3B, D), suggesting that Mec1 mediates the carbon source dependent Rnr3 induction independently of its DDR network.

The conserved ATP/dATP allosteric regulatory site facilitates efficient removal of Rnr1 in response to carbon source

Two lines of evidence suggest that the glycerol dependent Rnr1 down regulation might be via autophagy; (i) autophagy regulates Rnr1 abundance in response to genotoxic stress [22] and (ii) budding yeasts activate autophagy in response to non-fermentable carbon source [23]. We wished to test this possibility. First, we utilized GFP-ATG8 as a readout [23], to confirm the glycerol dependent autophagy in our experimental system. Results confirmed a marked increase in GFP, the autophagic cleavage product of the GFP-Atg8 in response to glycerol (Figure 4A). Next, we assessed the impact of atg1D. ATG1 encodes for the serine/threonine kinase subunit of the Atg1 signalling complex required for autophagy [24]. The mutation abolished glycerol dependent GFP accumulation, confirming that autophagy was impaired (Figure 4B). Crucially, Rnr1 persisted in the mutant, implicating autophagy in the down regulation (Figure 4B). To further confirm, we tested additional ATG genes; ATG5, 9, 13, 15, 16, and 18 required for the essential autophagosomal membrane formation, ATG17 and 31 for starvation induced autophagy, ATG32 for mitophagy, and ATG11, 19, and 24 for in cytoplasm-tovacuole (CVT) pathway [25]. Surprisingly, results show that the carbon source dependent Rnr1 down regulation proceeds normally in these mutants, indicating that the down regulation is not via autophagy (Figure 4C; data not shown).

Results above imply that the carbon source dependent down regulation of Rnr1 is mediated by an autophagy independent function of Atg1. ATG1 is notable among autophagy genes for its role(s) in facilitating mitochondrial respiration [26], which suggests that the latter might be involved. To address the possibility, we examined the impact of gut $2 \Delta$ and $n d i 1 \Delta$, which impair oxidative phosphorylation: Gut2 is an integral component of the mitochondrial outer membrane involved in glycerol metabolism and $\mathrm{NADH}$ oxidation [27]. Ndi1 is a mitochondrial protein that transfers electrons from NADH to ubiquinone in the respiratory chain [28]. Results show that the glycerol dependent Rnr1 down regulation is abolished in these mutants, con-
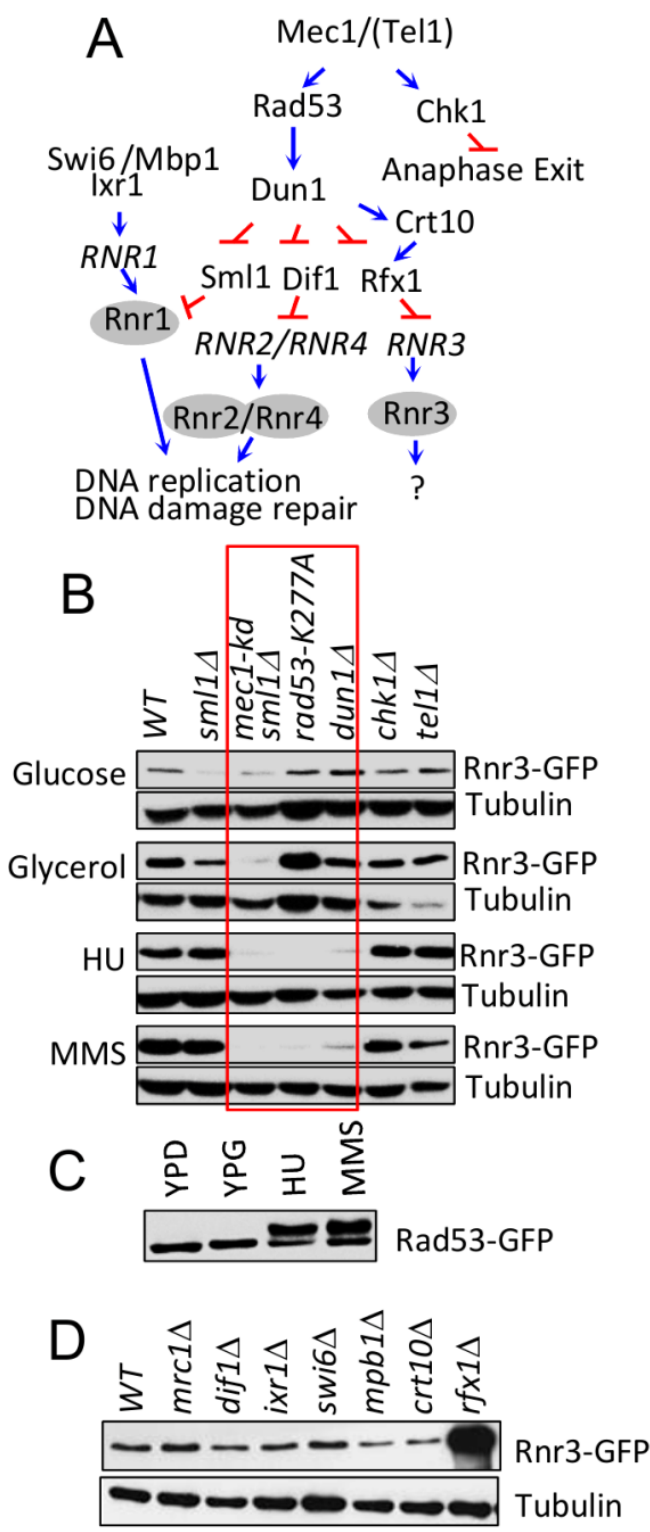

FIGURE 3: Mec1 mediates the carbon source dependent induction of Rnr3 independently of its DNA damage response network. (A) Key components of the Mec1 checkpoint response network involved in RNR regulation [19]. Blue arrows and red bars denote a positive and inhibitory impact on the indicated gene or protein. (B) Strains of the indicated genotypes (Table S1) were cultured in YPD, YPG, and YPD supplemented with $\mathrm{HU}(10 \mathrm{mM})$ or MMS (0.01\%). WCE samples were prepared from cells in log phase (YPD and YPG) or following 2 hours exposure to $\mathrm{HU}$ or MMS and subjected to Western blot analysis using $\alpha$-GFP antibodies. Tubulin was used as a loading control. Red box: Mutations that abolish the DDR dependent Rnr3 induction. (C) RAD53-GFP cells were cultured in YPD, YPG, and YPD supplemented with HU (10 $\mathrm{mM}$ ) or MMS (0.01\%). WCE samples were prepared from cells in log phase (YPD and YPG) or following 2 hours exposure to $\mathrm{HU}$ or MMS and subjected to Western blot analysis using $\alpha$ GFP antibodies. (D) WCE samples prepared from the indicated strains (Table S1) grown to mid-log phase in YPG were subjected to Western blot analysis using $\alpha$-GFP antibodies. Tubulin was used as a loading control. 
sistent with the notion that the down regulation is dependent on mitochondrial respiration (Figure 4D). We also assessed the impact of tor $1 \Delta$ and $\operatorname{rox} 1 \Delta$. Tor1 (Target of Rapamycin) is a serine/threonine kinase involved in nutrient response [29]. Rox1 is a transcription regulator involved in osmotic and hypoxic stress response [30]. Unlike the gut $2 \Delta$ and ndi1 $\Delta$ strains, tor $1 \Delta$ and $\operatorname{rox} 1 \Delta$ cells are proficient in glycerol dependent Rnr1 down regulation (Figure 4D), suggesting that the impact of glycerol is largely due to oxidative phosphorylation irrespective of other effects of the carbon source.

gut $2 \Delta$ and ndi1 $\Delta$ cells are also impaired the glycerol dependent Rnr3 induction; in contrast, both are proficient in $\mathrm{HU}$ or MMS dependent Rnr3 induction (Figure $4 \mathrm{~F}$ ). The latter provides further support for the proposal that the DDR- and carbon source-dependent Rnr3 induction is mediated by distinct mechanisms.

An essential function of oxidative phosphorylation is ATP production. Notably, ATP is a key regulator of RNR, whose binding to the conserved ATP/dATP allosteric site in the R1 subunit activates the enzyme [1]. This suggests that the effects of respiratory carbon source might be linked to the increased ATP production and its impact on the ATP/dATP binding site. To address this possibility, we ex- amined an allele where the ATP/dATP binding site has been mutated, the rnr1-D57N [31]. A notable delay in Rnr1 down regulation was observed in the mutant, consistent with the notion that the down regulation is dependent on ATP levels (Figure 4E). The latter also suggests that Rnr1 may function as a sensor for metabolic changes in the cell, facilitating its own down regulation.

\section{Novel genetic interactors implicate Rnr3's involvement in} cellular metabolism

Synthetic genetic array (SGA) analysis is a high throughput platform for identifying genetic interactors of a gene of interest; moreover, it is a powerful means to uncover a novel function(s) of the gene [32]. We examined the SGA database and found 130 genetic interactors of RNR3 (Table S2) [32]. Despite the fact that Rnr3 is a catalytic subunit of RNR, whose expression is induced in response to genotoxic stress, there was no enrichment of gene ontology (GO) terms directly linked to DNA synthesis or the DDR (Table S3). Instead, they were enriched for several extra nuclear processes such as "protein transport", "leucine catabolic process" and "cellular lipid metabolic processes" (Figure 5A; Tables S3).
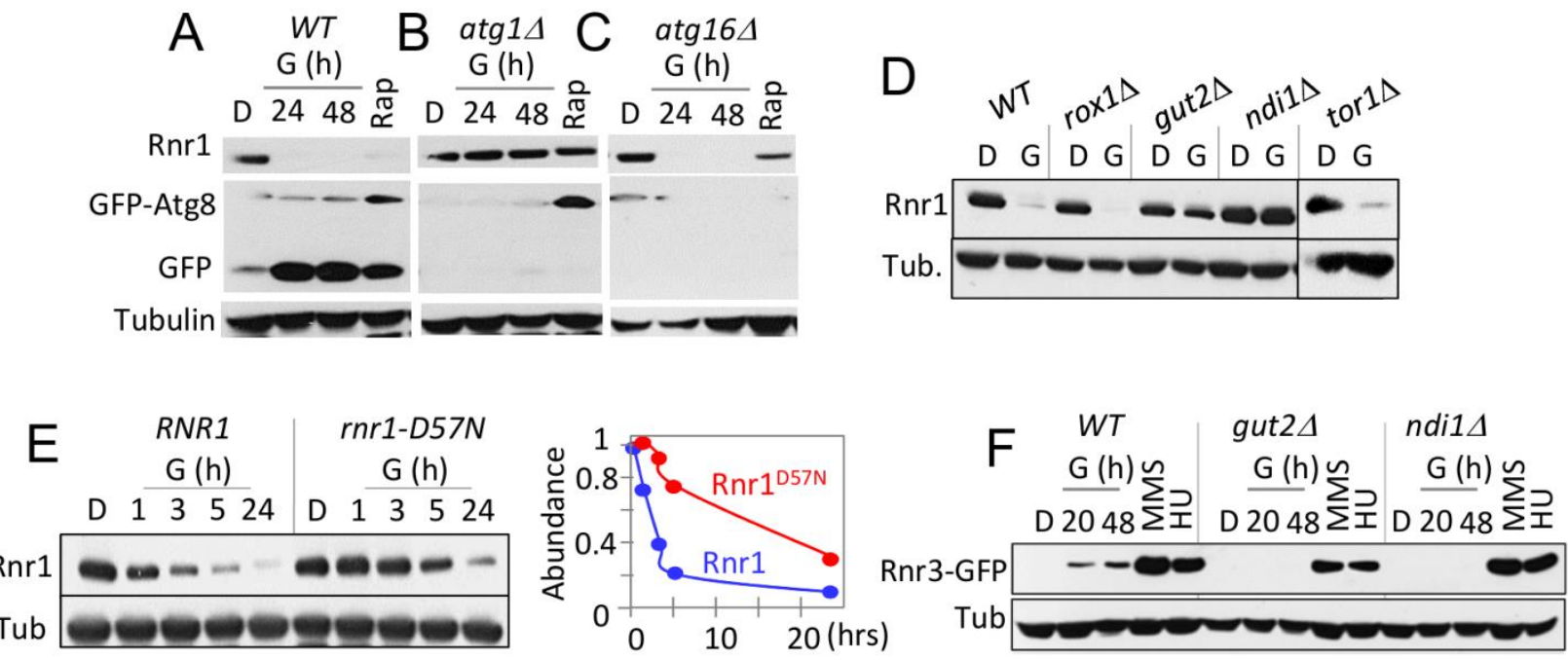

FIGURE 4: Carbon source dependent down regulation of Rnr1 is dependent on oxidative phosphorylation and the conserved ATP/dATP binding site. (A) WT cells transformed with a GFP-ATG8 plasmid was grown to mid log phase in leucine drop out medium supplemented with $2 \%$ glucose (SD-LEU; "D"). The cells were collected and transferred to leucine drop out medium supplemented with $2 \%$ glycerol (SGLEU; “G”) or SD-LUE2 supplemented with rapamycin ( $200 \mathrm{ng} / \mathrm{ml}$; “Rap"). WCE samples were prepared from the glycerol culture at 24 - and 48-hours after the medium switch or 2 hours in rapamycin, and subjected Western blot analysis using $\alpha$-Rnr 1 and $\alpha$-GFP antibodies. Tubulin was used as a loading control. (B, C) An atg1D - and atg16 - - strains transformed with the GFP-ATG8 plasmid were subjected to the analysis described in panel (A). (D) Strains of the indicated genotypes (Table S1) were grown to mid-log phase in YPD ("D") or YPG (“G"). WCE samples were prepared and subjected to Western blot analysis using $\alpha$-Rnr1 antibodies. Tubulin was used as a loading control. (E) WT and rnr1-D57N cells were grown to mid log phase in YPD ("D"). Cells were collected and released into YPG ("G") for further incubation. Left hand panel: Western blot analysis using $\alpha$-Rnr1 antibodies. Tubulin was used as a loading control. Right hand side: The Rnr1 signal in each lane was normalized to the tubulin signal in the same lane. The value obtained at each time point was expressed as a fraction of the value at $\mathrm{t}=0$. (F) WT, gut $2 \Delta$, and ndi1 $\Delta$ strains were cultured in YPD (“D”), YPG ("G"), and YPD supplemented with HU (10 mM) or MMS (0.01\%). WCE samples in YPD were from cells in mid log phase. The YPG samples were prepared at 20 and 48 hours after medium switch to YPG. The HU and MMS samples were prepared following 2 hour exposure to the respective chemical. WCE samples were subjected to Western blot analysis using $\alpha$-GFP antibodies. Tubulin was used as a loading control. 
Among the $R N R 3$ interactors associated with the GO term "protein transport" is TOM6 (Figure 5A; Table S2, S3). Tom6 is a component of the mitochondrial TOM (translocase of outer membrane) complex, responsible for mitochondrial protein import [33]. We find that tom $6 \Delta$ confers temperature sensitivity, which becomes more pronounced under respiratory conditions (Figure 5B; compare YPD versus YPG). Remarkably, $r n r 3 \Delta$ rescues the tom $6 \Delta$ phenotype, implicating a functional link between Rnr3 and mitochondria (Figure 5B). Notably, the rescue was observed under both respiratory (YPG) and fermentative conditions (YPD). This was unexpected given that Rnr3 is undetectable during fermentative proliferation (e.g. Figure 1A). We infer that Rnr3 is not only expressed under fermentative conditions, but also facilitates an essential mitochondrial function(s).

In budding yeast, mitochondrial DNA is dispensable under fermentative conditions [35]. Therefore, the $r n r 3 \Delta$ rescue of tom $6 \Delta$ growth defects, which manifests under both fermentative and respiratory conditions, suggests that the rescue is independent of mitochondrial genome maintenance and, by extension, dNTP production. To confirm, we assessed the impact of an allele, rnr3-C428A, car- rying a mutation at a conserved cysteine residue in the active site. The corresponding mutation in Rnr1, the rnr1$C 428 A$, is a catalytically dead allele [36]. We introduced the rnr3-C428A plasmid marked with LEU2 into a WT and rnr3A strains and assessed the impact in leucine drop out medium supplemented with either $2 \%$ glucose (SD-LEU) or $2 \%$ glycerol (SG-LEU) (Figure 5C). Surprisingly, we find that $r n r 3 \Delta$ cells on leucine drop out plates, unlike the YP plates, exhibit a notable growth defects in glycerol (Figure 5C) While the impact of leucine drop-out medium was unexpected, it is consistent with the enrichment of genes associated with the GO term "leucine catabolic process" among the $R N R 3$ genetic interactors (Figure $\mathbf{5 A}$ ).

The glycerol sensitivity of $r n r 3 \Delta$ cells was exacerbated

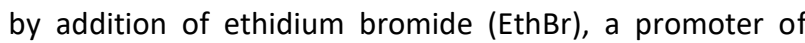
mitochondrial genome loss, and elevated temperature (Figure $\mathbf{5 C}$ ). Introduction of $R N R 3$ rescued the phenotype confirming that it was due to the absence of Rnr3 (Figure 5C). Importantly, the rnr3-C428A plasmid also rescued the phenotype (Figure $\mathbf{5 C}$ ), in support for the proposal that the impact of Rnr3 under respiratory conditions is independent of dNTP.

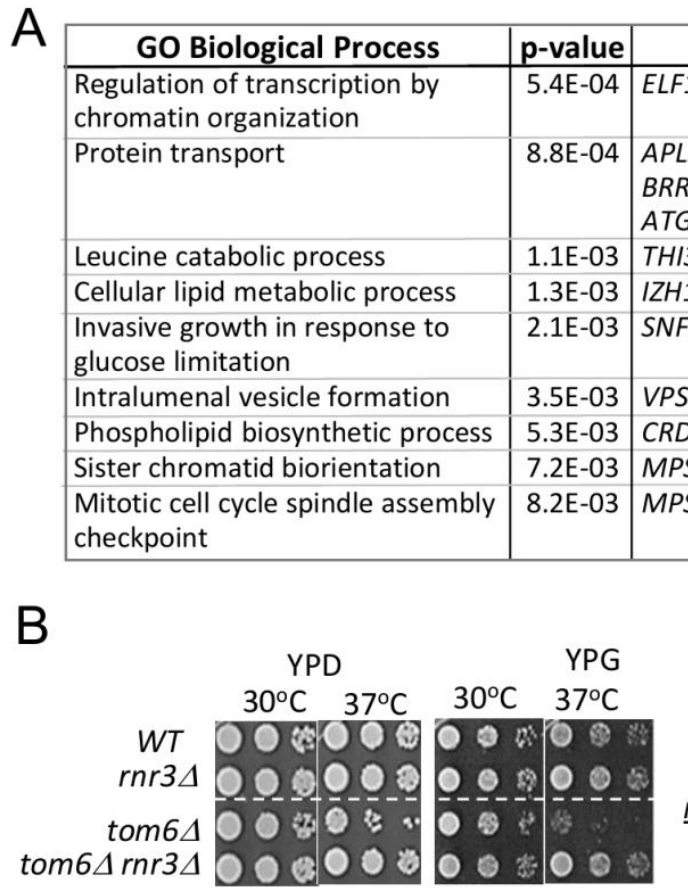

RNR3 Interactors LF1 HDA1 RPD3

APL3 KAP104 CIS1 PEX7 VPS64 BST1 BRR6 VPS62 APL6 APS2 MOG1 VPS20 ATG34 TOM6 ARF3 COG4 VPS4 PDI

ZH1 ROG1 BRR6 VPS20 VPS4 CRD1 PCT1 PSD1 INO4 MPS1 SPC105 MPS1 SPC105 BUB3

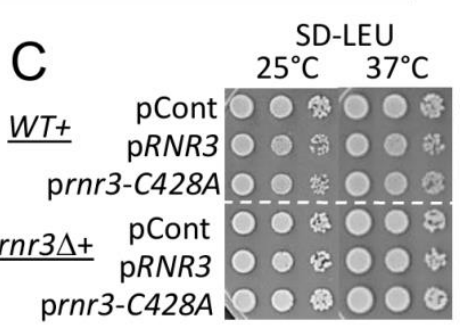

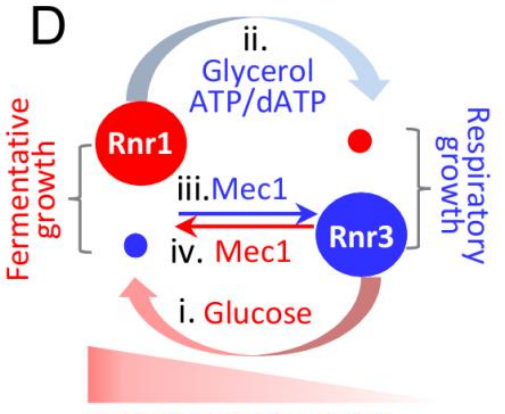

Rnr1: Demand for dNTPs

Rnr3: Mitochondrial function

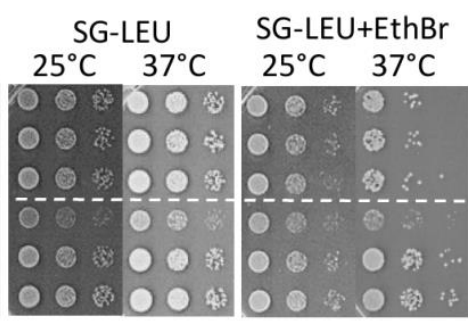

FIGURE 5: Novel genetic interactors unveil a functional link between Rnr3 and mitochondria. (A) The SGA database lists 130 genetic interactors of RNR3 (Tables S2) [32]. Functional Specification (FunSpec) analysis [39] unveils enrichment of several GO Biological Process terms (Tables S3). (B) Ten fold serial dilutions of the indicated strains were spotted onto YPD or YPG and incubated at the indicated temperatures. (C) WT and $r n r 3 \Delta$ cells transformed with a RNR3-, rnr3-C428A-, or an empty LEU2 vector plasmid were spotted onto SD-LEU, SGLEU, or SG-LEU plus EthBr $(0.5 \mu \mathrm{g} / \mathrm{ml})$ and incubated at the indicated temperatures. (D) Model: Mec1 and carbon source dependent regulation of Rnr1 and Rnr3 facilitates fermentative and respiratory growth. (i) Abundant glucose induces Rnr1 in preparation for rapid proliferation and a greater demand for dNTPs. (ii) Glucose depletion or non fermentable carbon sources down regulate Rnr1 as a means to attenuate dNTP production in preparation for slowed growth under respiratory conditions. (iii) Mec1 mediates the respiratory carbon source dependent induction of Rnr3 independently of its DDR network. (iv) Mec1 also facilitates the glucose dependent down regulation of Rnr3 via a mechanism yet to be elucidated. 


\section{DISCUSSION}

We present evidence for a carbon source dependent regulation of the budding yeast RNR that impacts expression of the two catalytic subunits, Rnr1 and Rnr3. Nonfermentable carbon sources or limiting concentrations of glucose down regulate Rnr1 and induce Rnr3 expression. Oppositely, abundant glucose induces Rnr1 expression and down regulates Rnr3. While the mechanistic basis and physiological significance are yet to be elucidated, results above provide several insights.

Mec1, an essential ATM/ATR checkpoint response kinase, mediates both the respiratory carbon source dependent induction and glucose dependent removal of Rnr3. Surprisingly, Mec1 mediates the effects independently of the key known components of its DDR network, including Rad53 and Dun1. Our findings implicate a novel Mec1 signalling axis.

We present three lines of evidence for involvement of Rnr3 in cellular metabolism; (i) Analysis of RNR3 genetic interactors in the SGA database unveils enrichment of genes linked to metabolic processes (Figure 5A; Table S3). (ii) $r n r 3 \Delta$ confers respiratory growth defects when cultured in leucine drop out medium (Figure 5C). And (iii) $r n r 3 \Delta$ rescues temperature sensitivity of tom6 $\Delta$ (Figure $5 \mathrm{~B}$ ). The latter two suggest a role of Rnr3 in mitochondrial function. In support, two independent studies on mitochondrial proteome analyses found that Rnr3 localizes to the organelle $[37,38]$.

Deletion of $R N R 3$ rescues tom6 $\Delta$ phenotype under both respiratory and fermentative conditions, indicating that the rescue is independent of mitochondrial genome maintenance (Figure 5B). And a rnr3 allele carrying a mutation at a critical cysteine residue in the active site, $r n r 3-$ C428A, recues respiratory growth defects of $r n r 3 \Delta$ (Figure 5C). Together, these results suggest that Rnr3 has a dNTP independent mitochondrial function. This is consistent with the findings that Rnr3 is the sole component of the yeast RNR found in mitochondria [37, 38].

Three lines of evidence suggest that the respiratory of carbon source dependent down regulation of Rnr1 is linked to oxidative phosphorylation and the ensuing changes in intracellular ATP/dATP ratio: (i) ATG1, involved in activation of mitochondrial respiration, is the only ATG gene among the tested to be required for Rnr1 down regulation. (ii) Mutations that impair oxidative phosphorylation, gut2 $\Delta$ and $n d i 1 \Delta$, abolish the down regulation. And (iii) down regulation of $\mathrm{Rnr} 1^{\mathrm{D} 57 \mathrm{~N}}$, carrying a mutation at the conserved ATP/dATP allosteric regulatory site, is markedly delayed. Intriguingly, the latter suggests that Rnr1 may function as a sensor for changes in metabolic state via its ATP/dATP binding site.

The dramatic reduction in Rnr1 abundance suggests that the gene might be dispensable under respiratory conditions. On the contrary, we find that $r n r 1 \Delta$ spores from a $r n r 1 \Delta /+$ diploid strains are unable to form a colony on YPG plate (data not shown). We infer that that Rnr1 is expressed under respiratory conditions at a low level to promote the essential dNTP production.
Taken together, we propose the following model (Figure 5D): The carbon source dependent regulation of Rnr1 is a mechanism to control RNR activity to couple dNTP production to differential demands for the DNA building blocks. In the presence of abundant glucose, which facilitates rapid fermentative proliferation, Rnr1 is induced to meet the increased demand for dNTPs. In response to a non-fermentable carbon source or glucose depletion, which activates slower respiratory proliferation, Rnr1 level drops dramatically to prevent accumulation of excessive dNTPs, which can lead to mutator phenotype and/or hinder cell cycle progression [31]. The carbon source dependent regulation of $\mathrm{Rnr} 3$, on the other hand, is a mechanism to activate a dNTP independent function of Rnr3, which becomes more critical under respiratory conditions. Mec1, an essential ATM/ATR protein kinase, mediates both the carbon source dependent induction and removal of Rnr3, independently of its DDR network (Figure 5D). These findings provide a new framework in understanding the function(s) of Rnr3 and Mec1.

\section{MATERIALS AND METHODS \\ Yeast strains and media}

All strains were of either BY or S288C background, except for mec1-kd RNR3-GFP and rad53-K277A RNR3-GFP, which were generated via crossing a BY and SK1 strain (Table S1). Synthetic complete (SC) media were prepared as described [40] with one of the following carbon sources; $2 \%(\mathrm{w} / \mathrm{v})$ glucose, $0.1 \%$ $(\mathrm{w} / \mathrm{v})$ glucose, $2 \%(\mathrm{v} / \mathrm{v})$ glycerol, or $3 \%(\mathrm{v} / \mathrm{v})$ ethanol. $\mathrm{HU}$ and MMS media were YPD ( $1 \%$ yeast extract, $2 \%$ bacto peptone, $2 \%$ glucose) supplemented with $10 \mathrm{mM} \mathrm{HU}$ or $0.01 \%(\mathrm{v} / \mathrm{v})$ MMS, respectively.

\section{Yeast cultures}

All liquid cultures were incubated at $30^{\circ} \mathrm{C}$ unless noted. For a carbon source switching experiment, a $5 \mathrm{ml}$ overnight culture in $2 \%$ glucose was diluted to $\mathrm{OD}_{600}$ of 0.2 or less on the following morning. The culture was incubated further for $\sim 5$ hours until the $\mathrm{OD}_{600}$ reached $\sim 0.5$. Cells were washed once in $\mathrm{H}_{2} \mathrm{O}$ and resuspended in the medium of choice and incubated further. For glycerol-, ethanol-, or $0.1 \%$ glucose cultures, the volume was kept at $20 \%$ of the flask size (e.g. $50 \mathrm{ml}$ culture in a $250 \mathrm{ml}$ flask) and shaken at $180 \mathrm{rpm}$ to ensure adequate aeration.

\section{rnr3-C428A plasmid construction}

For the construction of the plasmids $p R N R 3$ and $p R N R 3-C 428 A$, the RNR3 ORF ( $\pm 500 \mathrm{bp}$ ) was amplified by PCR using genomic DNA from a $r n r 1 \Delta$ mutant as a template. The primers used for the amplification of the endogenous $R N R 3$ include sequences upstream and dowstream of the RNR3 ORF (bold) and sequences complementary to the vector pRS315, upstream the Pst1 restriction site (Primer A) and downstream the HindlII restriction site (Primer B) (Primer A: 5'GGCGGCCGCTCTAGAACTAGTGGATCCCCCGGGCTGCAGG GGCT TGTTTCAGTTTGAACT-3' and Primer B: 5'-ACCGGG CCCCCCCTCGAGGTCGACGGTATCGATAAGCTTG AATTCAATGCT AAATGGTC-3'). For the construction of $\mathrm{p} R N R 3-C 428 A$, primers containing the mutation C428A were designed (Primer C: $5^{\prime}$ ATTCGACGATTTCACAGGCTAAATTAGATGATTTGAT-3', and Primer D: 5'-ATCAAATCATCTAATTTAGCCTGTGAA ATCGTC 
GAAT-3'). The amplification of the rnr3 allele carrying the C468A alteration was carried out using the combination of Primers A and C, and Primers B and D. The cloning of the amplified sequences into the plasmid was mediated by gap repair in yeast: For the construction of $\mathrm{p} R N R 3$, a $r n r 3 \Delta$ strain was transformed with the Pst1/HindIII digested pRS315 and the $R N R 3$ fragment amplified using primers $\mathrm{A}$ and $\mathrm{B}$. For the construction of prnr3-C428A, the rnr3-C428A fragment amplified using primers $C$ and $D$ were transformed instead. Transformants were selected in leucine drop out synthetic media. Plasmids from the transformants were extracted, amplified in E. coli, and the introduction of the mutation was confirmed by DNA sequencing analysis.

\section{Spot test}

Ten-fold serial dilutions from a mid-log culture $\left(10^{7}\right.$ cells $\left./ \mathrm{ml}\right)$ were prepared and spotted onto indicated agar plates. Once dry, the agar plates were incubated at the indicated temperature for $1-3$ day ( $2 \%$ glucose) or $4-7$ ( $2 \%$ glycerol) days before images were taken.

\section{Western blot analysis}

Western blot analysis was performed on TCA (trichloroacetic acid) extracts prepared from culture volumes corresponding to $10 \mathrm{OD}_{600}$ units. Protein extracts were precipitated and pellets were resuspended in loading buffer. After boiling, samples were loaded on $7.5 \%$ polyacrylamide gels. GFP tagged proteins (Rnr3, Rnr2, Rnr4, Rad53) were detected with mouse $\alpha$-GFP antibody (Roche 11814460001, 1:5000). Rnr1 was detected with rabbit $\alpha$-Rnr1 antibody (Agrisera AS16 3639, 1:10000). Rabbit $\alpha$-tubulin antibody was used for the detection of tubulin, our loading control (Abcam ab184970, 1:20000). Second-

\section{REFERENCES}

1. Nordlund P, and Reichard P (2006). Ribonucleotide Reductases. Annu Rev Biochem 75(1): 681-706. doi: 10.1146/annurev.biochem.75.103004.142443

2. Chabes a, Domkin V, Larsson G, Liu a, Graslund a, Wijmenga S, and Thelander $L$ (2000). Yeast ribonucleotide reductase has a heterodimeric iron-radical-containing subunit. Proc Natl Acad Sci U S A 97(6): 2474-9. doi: 10.1073/pnas.97.6.2474

3. Elledge SJ, Zhou Z, and Allen JB (1992). Ribonucleotide reductase: regulation, regulation, regulation. Trends Biochem Sci 17(3): 119-123. doi: 10.1016/0968-0004(92)90249-9

4. Zhao X, and Rothstein R (2002). The Dun1 checkpoint kinase phosphorylates and regulates the ribonucleotide reductase inhibitor Sml1. Proc Natl Acad Sci U S A 99(6): 3746-51. doi: 10.1073/pnas.062502299

5. Harper JW, and Elledge SJ (2007). The DNA Damage Response: Ten Years After. Mol Cell 28(5): 739-745. doi: 10.1016/j.molcel.2007.11.015

6. Carballo JA, Panizza S, Serrentino M, Johnson AL, Geymonat M, Borde V, Klein F, and Cha RS (2013). Budding yeast ATM/ATR control meiotic double-strand break (DSB) levels by down-regulating Rec114, an essential component of the DSB-machinery. PLoS Genet 6: e1003545. doi: 10.1371/journal.pgen.1003545

7. Cha RS, and Kleckner N (2002). ATR homolog Mec1 promotes fork progression, thus averting breaks in replication slow zones. Science 297(5581): 602-606. doi: 10.1126/science.1071398

8. Corcoles-Saez I, Dong K, Waskiewicz E, Costanzo M, Boone C, and ary antibodies used were goat $\alpha$-Mouse IgG H\&L (Abcam Ab6789, 1:10000) and $\alpha$-Rabbit IgG (Cell Signalling \#7074, 1:5000). Secondary antibodies were detected using Western Lightning ECL Pro (PerkinElmer).

\section{ACKNOWLEDGMENTS}

The authors thank Yoshinori Ohsumi and Andrei Chabes for strains and plasmid. This work was supported by grants from North West Cancer Research to R.C. (CR961 and CR1161).

\section{SUPPLEMENTAL MATERIAL}

All supplemental data for this article are available online at www.microbialcell.com.

\section{CONFLICT OF INTEREST}

The authors declare no conflicting interests.

\section{COPYRIGHT}

(C) 2019 Corcoles-Saez et al. This is an open-access article released under the terms of the Creative Commons Attribution (CC BY) license, which allows the unrestricted use, distribution, and reproduction in any medium, provided the original author and source are acknowledged.

Please cite this article as: Isaac Corcoles-Saez, Jean-Luc Ferat, Michael Costanzo, Charles M. Boone and Rita S. Cha (2019). Functional link between mitochondria and Rnr3, the minor catalytic subunit of yeast ribonucleotide reductase. Microbial Cell 6(6): 286-294. doi: 10.15698/mic2019.06.680

Cha RS (2018). Essential Function of Mec1, the Budding Yeast ATM/ATR Checkpoint-Response Kinase, in Protein Homeostasis. Dev Cell 46: 495-503. doi: 10.1016/j.devcel.2018.07.011

9. Cheng A, Zhao T, Tse K-H, Chow H-M, Cui Y, Jiang L, Du S, Loy MMT, and Herrup K (2018). ATM and ATR play complementary roles in the behavior of excitatory and inhibitory vesicle populations. Proc Natl Acad Sci 115(2): E292-E301. doi: 10.1073/pnas.1716892115

10. Lee JH, Mand MR, Kao CH, Zhou Y, Ryu SW, Richards AL, Coon JJ, and Paull TT (2018). ATM directs DNA damage responses and proteostasis via genetically separable pathways. Sci Signal 11(512): 10.1126/scisignal.aan5598. doi: 10.1126/scisignal.aan5598

11. Zhao X, Chabes a, Domkin V, Thelander L, and Rothstein R (2001) The ribonucleotide reductase inhibitor Sml1 is a new target of the Mec1/Rad53 kinase cascade during growth and in response to DNA damage. EMBO J 20(13): 3544-3553. doi: 10.1093/emboj/20.13.3544

12. Blackford AN, and Jackson SP (2017). ATM, ATR, and DNA-PK: The Trinity at the Heart of the DNA Damage Response. Mol Cell 66(6): 801-817. doi: 10.1016/j.molcel.2017.05.015

13. Zhou Z, and Elledge SJ (1993). DUN1 encodes a protein kinase that controls the DNA damage response in yeast. Cell 75(6): 1119-1127. doi: 10.1016/0092-8674(93)90321-G

14. Lee YD, Wang J, Stubbe J, and Elledge SJ (2008). Dif1 Is a DNADamage-Regulated Facilitator of Nuclear Import for Ribonucleotide Reductase. Mol Cell 32(1): 70-80. doi: 10.1016/j.molcel.2008.08.018

15. Elledge $S$, and Davis $R$ (1990). Two genes differentially regulated in the cell cycle and by DNA-damaging agents encode alternate 
regulatory subunits of ribonucleotide reductase. Genes Dev 4: 740751. doi: 10.1101/gad.4.5.740

16. Byrne KP, and Wolfe KH (2005). The Yeast Gene Order Browser: Combining curated homology and syntenic context reveals gene fate in polyploid species. Genome Res 15(10): 1456-1461. doi: 10.1101/gr.3672305

17. Conant GC, and Wolfe KH (2007). Increased glycolytic flux as an outcome of whole-genome duplication in yeast. Mol Syst Biol 3: 129. doi: $10.1038 / \mathrm{msb} 4100170$

18. Paciotti V, Clerici M, Scotti M, Lucchini G, and Longhese MP (2001) Characterization of mec1 kinase-deficient mutants and of new hypomorphic mec1 alleles impairing subsets of the DNA damage response pathway. Mol Cell Biol 21(12): 3913-3925. doi: 10.1128/MCB.21.12.3913-3925.2001

19. Tsaponina O, Barsoum E, Åström SU, and Chabes A (2011). Ixr1 is required for the expression of the ribonucleotide reductase Rnr1 and maintenance of dNTP pools. PLoS Genet 7(5). doi: 10.1371/journal.pgen.1002061

20. Craven RJ, Greenwell PW, Dominska M, and Petes TD (2002). Regulation of genome stability by TEL1 and MEC1, yeast homologs of the mammalian ATM and ATR genes. Genetics 161(2): 493-507. PMID: 12072449

21. Sanchez Y, Bachant J, Wang H, Hu F, Liu D, Tetzlaff M, and Elledge SJ (1999). Control of the DNA Damage Checkpoint by Chk1 and Rad53 Protein Kinases Through Distinct Mechanisms. Science 286(5442): 1166-1171. doi: 10.1126/science.286.5442.1166

22. Dyavaiah M, Rooney JP, Chittur S V, Lin Q, and Begley TJ (2011). Autophagy-dependent regulation of the DNA damage response protein ribonucleotide reductase 1 . Mol Cancer Res 9(4): 462-475. doi: 10.1158/1541-7786.MCR-10-0473

23. Okamoto K, Kondo-Okamoto N, and Ohsumi Y (2009). Mitochondria-Anchored Receptor Atg32 Mediates Degradation of Mitochondria via Selective Autophagy. Dev Cell 17(1): 87-97. doi: 10.1016/j.devcel.2009.06.013

24. Nakatogawa H, Suzuki K, Kamada $Y$, and Ohsumi $Y$ (2009). Dynamics and diversity in autophagy mechanisms: lessons from yeast. Nat Rev Mol Cell Biol 10(7): 458-467. doi: 10.1038/nrm2708

25. Nakatogawa H, Suzuki K, Kamada Y, and Ohsumi $Y$ (2009). Dynamics and diversity in autophagy mechanisms: lessons from yeast. Nat Rev Mol Cell Biol 10(7): 458-467. doi: 10.1038/nrm2708

26. Yi C, Tong J, Lu P, Wang Y, Zhang J, Sun C, Yuan K, Xue R, Zou B, Li $\mathrm{N}$, Xiao S, Dai C, Huang Y, Xu L, Li L, Chen S, Miao D, Deng H, Li H, and Yu $L$ (2017). Formation of a Snf1-Mec1-Atg1 Module on Mitochondria Governs Energy Deprivation-Induced Autophagy by Regulating Mitochondrial Respiration. Dev Cell 41(1): 59-71.e4. doi: 10.1016/j.devcel.2017.03.007

27. Ronnow B, and Kielland-Brandt MC (1993). GUT2, a gene for mitochondrial glycerol 3-phosphate dehydrogenase of Saccharomyces cerevisiae. Yeast 9(10): 1121-1130. doi: 10.1002/yea.320091013
28. Marres CA, de Vries S, and Grivell LA (1991). Isolation and inactivation of the nuclear gene encoding the rotenone-insensitive internal $\mathrm{NADH}$ : ubiquinone oxidoreductase of mitochondria from Saccharomyces cerevisiae. Eur J Biochem 195(3): 857-862. doi: 10.1111/j.1432-1033.1991.tb15775.x

29. Cardenas ME, Cutler NS, Lorenz MC, Di Como CJ, and Heitman J (1999). The TOR signaling cascade regulates gene expression in response to nutrients. Genes Dev 13(24): 3271-3279. doi: 10.1101/gad.13.24.3271

30. Lowry C V, and Zitomer RS (1984). Oxygen regulation of anaerobic and aerobic genes mediated by a common factor in yeast. Proc Natl Acad Sci U S A 81(19): 6129-6133. doi: 10.1073/pnas.81.19.6129

31. Chabes A, and Stillman B (2007). Constitutively high dNTP concentration inhibits cell cycle progression and the DNA damage checkpoint in yeast Saccharomyces cerevisiae. Proc Natl Acad Sci U S A 104(4): 1183-8. doi: 10.1073/pnas.0610585104

32. Costanzo $\mathrm{M}$ et al. (2016). A global genetic interaction network maps a wiring diagram of cellular function. Science 353(6306): aaf1420-aaf1420. doi: 10.1126/science.aaf1420

33. Neupert W, and Herrmann JM (2007). Translocation of Proteins into Mitochondria Translocase: a membrane- embedded protein complex that mediates translocation of polypeptides from one side of the membrane to the other side. Annu Rev Biochem doi: 10.1146/annurev.biochem.76.052705.163409

34. Wiesenberger G, and Fox TD (1997). Pet127p, a membraneassociated protein involved in stability and processing of Saccharomyces cerevisiae mitochondrial RNAs. Mol Cell Biol 17(5): 2816-2824. doi: 10.1128/mcb.17.5.2816

35. Chen XJ, and Clark-Walker GD (1999). The petite mutation in yeasts: 50 years on. Int Rev Cytol 194: 197-238. doi: 10.1016/S00747696(08)62397-9

36. Domkin V, Thelander L, and Chabes A (2002). Yeast DNA damageinducible Rnr3 has a very low catalytic activity strongly stimulated after the formation of a cross-talking Rnr1/Rnr3 complex. J Biol Chem 277(21): 18574-18578. doi: 10.1074/jbc.M201553200

37. Sickmann A, Reinders J, Wagner $Y$, Joppich C, Zahedi R, Meyer HE, Schönfisch B, Perschil I, Chacinska A, Guiard B, Rehling P, Pfanner N, and Meisinger $C$ (2003). The proteome of Saccharomyces cerevisiae mitochondria. Proc Natl Acad Sci U S A 100(23): 13207-12. doi: 10.1073/pnas. 2135385100

38. Reinders J, Zahedi RP, Pfanner N, Meisinger C, and Sickmann A (2006). Toward the complete yeast mitochondrial proteome: Multidimensional separation techniques for mitochondrial proteomics. J Proteome Res 5(7): 1543-1554. doi: 10.1021/pr050477f

39. Robinson MD, Grigull J, Mohammad N, and Hughes TR (2002). FunSpec: a web-based cluster interpreter for yeast. BMC Bioinformatics 3: 35. doi: 10.1186/1471-2105-3-35

40. Tong AHY, and Boone C (2007). Yeast Gene Analysis - Second Edition. Methods Microbiol 36: 369-707. doi: 10.1016/S05809517(06)36016-3 